\title{
REDUCTION OF DISCRETE ALGEBRAIC RICCATI EQUATIONS: ELIMINATION OF GENERALIZED EIGENVALUES ON THE UNIT CIRCLE
}

\author{
Augusto FERRAnTE AND LOREnZO NTOGRAMATZIDIS
}

Abstract. The purpose of this paper is to introduce a two-stage procedure that can be used to decompose a discrete-time algebraic Riccati equation into a trivial part, a part that is entirely arbitrary, and a part that can be obtained by computing the set of solutions of a reduced-order Riccati equation whose associated symplectic pencil has no generalized eigenvalues on the unit circle.

Mathematics subject classification (2010): 93C05, 93B27, 93B52.

Keywords and phrases: Discrete-time algebraic Riccati equations, symplectic pencil.

\section{REFERENCES}

[1] H. Abou-Kandil, G. Freiling, V. Ionescu and G. Jank, it Matrix Riccati Equations in Control and Systems Theory, Birkhäuser, Basel, 2003.

[2] D. J. Clements ANd B .D. O. Anderson, Polynomial factorization via the Riccati equation, SIAM Journal on Applied Mathematics, 31 (2004), 179-205.

[3] T. DAMm AND D. HinRICHSEN, Newton's method for a rational matrix equation occurring in stochastic control, Linear Algebra and its Applications, 332-334 (2001), 81-109.

[4] A. FERRANTE, On the structure of the solution of discrete-time algebraic Riccati equation with singular closed-loop matrix, IEEE Transactions on Automatic Control, 49 (2004), 2049-2054.

[5] A. Ferrante And L. NTOgRamatzidis, The generalized discrete algebraic Riccati equation in Linear-Quadratic optimal control, Automatica, 49 (2013), 471-478.

[6] A. FerRante AND L. NTOgRamatzidis, The extended symplectic pencil and the finite-horizon $L Q$ problem with two-sided boundary conditions, IEEE Transactions on Automatic Control, 58 (2013), 2102-2107.

[7] A. Ferrante And L. NTOgRamatzidis, A note on finite-horizon LQ problems with indefinite cost, Automatica, 52 (2015), 290-293.

[8] A. FerRante And L. NTOGRAmatzidis, Generalized finite-horizon linear-quadratic optimal control, Encyclopedia of Systems and Control, DOI 10.1007/978-1-4471-5102-9_202-1, SpringerVerlag, London, 2014.

[9] A. FerRAnte AND H. K. Wimmer, Order reduction of discrete-time algebraic Riccati equations with singular closed-loop matrix, Operators and Matrices, 1 (2007), 61-70.

[10] G. Freiling AND A. HochHaus, On a class of rational matrix differential equations arising in stochastic control, Linear Algebra and its Applications, 379 (2004), 43-68.

[11] T. FuJinaka AND M. ARAKI, Discrete-time optimal control of systems with unilateral time-delays, Automatica, 23 (1987), 763-765.

[12] T. Fujinaka, G. Chen And H. Shibata, Discrete algebraic Riccati equation with singular coefficient matrix, In Proc. Systems and Networks: Mathematical Theory and Application (MTNS 98), Padova, Italy, 1999.

[13] A. HansSon And P. HagAnder, How to decompose semi-definite discrete-time algebraic Riccati equations, European Journal of Control, 5 (1999), 245-258.

[14] V. Ionescu, C. OARǍ And M. Weiss, Generalized Riccati theory and robust control, a Popov function approach, Wiley, 1999. 
[15] P. Lancaster And L. Rodman, Algebraic Riccati equations, Clarendon Press, Oxford, 1995.

[16] J. MedAnIC, Geometric properties and invariant manifolds of the Riccati equation, IEEE Transactions on Automatic Control, 27 (1985), 670-677.

[17] T. MITA, Optimal digital feedback control systems counting computation time of control laws, IEEE Transactions on Automatic Control, 30 (1985), 542-548.

[18] L. NTOGRAmATZIDIS AND A. FerRANTE, The discrete-time generalized algebraic Riccati Equation: order reduction and solutions's structure, Systems \& Control Letters, 75 (2015), 84-93.

[19] D. RAPPAPORT AND L. M. Silverman, Structure and stability of discrete-time optimal systems, IEEE Transactions on Automatic Control, 16 (1971), 227-233.

[20] W. T. REID, Riccati differential equations, Academic Press, 1972.

[21] J. C Willems, S. BitTanti And A. Laub, editors, The Riccati Equation, Springer Verlag, New York, 1991.

[22] H. K. WIMMER, Normal forms of symplectic pencils and the discrete-time algebraic Riccati equation, Linear Algebra and its Applications, 147 (1991), 411-440.

[23] M. ZoRZI, Robust Kalman filtering under model perturbations, IEEE Transactions on Automatic Control, 62, 6 (2017), 2902-2907.

[24] B. C. LEVY, M. ZORZI, A contraction analysis of the convergence of risk-sensitive filters, SIAM Journal on Control and Optimization, 54, 4 (2016), 2154-2173.

[25] M. ZoRZI, Convergence analysis of a family of robust Kalman filters based on the contraction principle, SIAM Journal on Control and Optimization, 55, 5 (2017), 3116-3131. 